\title{
The Usefulness of Current Perception Threshold Test in Both Lower Extremities with Diabetic Patient
}

\author{
DongwoOK Han, PhD, $\mathrm{PT}^{1)}$, MisoOK Ha, PhD, $\mathrm{PT}^{2)}$ \\ 1) Department of Physical Therapy, College of Medical and Life Science, Silla University \\ ${ }^{2)}$ Department of Physical Therapy, Choonhae College of Health Sciences: Ungchon-myeon, Ulju-gun, \\ Ulsan, 689-784 Republic of Korea. TEL: +82 52-270-0342, FAX: +82 52-270-0199, E-mail: \\ harpt@hanmail.net
}

\begin{abstract}
Purpose] The purpose of this study was to investigate the differences of sensory nerve fiber degeneration rates with duration of diabetes mellitus, as well as the difference in the degeneration rate of nerve fibers by selectively providing stimulation to the A-beta, A-delta, C fibers and measuring the current perception threshold (CPT) of diabetic patients. [Subjects] The subjects of this study were diabetic patients complaining of significant neuropathy symptoms such as numbness, dullness and paraesthesia. [Methods] Stimulations of $5 \mathrm{~Hz}, 250 \mathrm{~Hz}$, and 2,000 Hz of frequency were generated with a Neurometer CPT (Neurotron Inc., Baltimore, MD, U.S.A.) and delivered selectively to C fibers, Adelta fibers and A-beta fibers. The intensity of the stimulation of $5 \mathrm{~Hz}, 250 \mathrm{~Hz}$ and $2,000 \mathrm{~Hz}$ increased to $0.01 \mathrm{~mA}$. The independent t-test was used to compare perception thresholds. [Results] In the case of the right lower limb, the threshold value of A-beta fibers varied with the duration of diabetes and was particularly higher in the group of patients with 10 years or longer duration. The threshold value of A-delta fibers also varied with diabetes duration. That of the group with diabetes duration from 5 years to less than 10 years was not significantly different from that group with diabetes of the duration of 10 years or longer. However, the threshold value of the group with diabetes duration of 10 years or longer was higher than that of the groups of other durations. [Conclusion] The perception thresholds varied with diabetes duration and showed that degeneration of A-beta fibers and A-delta fibers that have myelin was more rapid than that of $\mathrm{C}$ fibers that are without myelin. Therefore, therapeutic interventions should be applied at the early stage of neuropathy.
\end{abstract}

Key words: Diabetes mellitus, Current Perception Threshold, Electrical Nerve Stimulation

(This article was submitted Apr. 27, 2010, and was accepted Jun. 10, 2010)

\section{INTRODUCTION}

Among Korean neuropathy cases, $30.8 \%$ of patients have diabetic neuropathy and the number of diabetic neuropathy patients abruptly increases over the age of forty ${ }^{1}$. Generally, as diabetes mellitus progresses the occurrences of diabetic neuropathy increases. If abnormality in nerve conduction studies without subjective symptoms is included, neuropathy is found in almost $100 \%$ of the diabetic patients ${ }^{2}$.

Neuropathy, one of the most common complications of diabetes mellitus (DM), shows symptoms caused by sensory nerve abnormality at an early stage ${ }^{3)}$. As the disease progresses, it invades to the motor nerve and manifests as muscle weakness or muscle atrophy. The clinical symptoms include hyposensitivity, dysesthesia, pain and loss of DTR (deep tendon reflex $)^{4)}$. Pain is a common problems for patients in rehabilitation. Although inquiry and physical examination according to symptoms are the most important findings for a diagnosis of neuropathy, they cannot be used at an advanced stage of the disease or for follow-up because they have tests that are very subjective and qualitative ${ }^{5)}$. In addition, a nerve conduction study sometimes provides normal readings even though there is diabetic neuropathy, which indicates a high probability of losing time for interventions by physical therapy ${ }^{6}$. . Therefore, the current perception threshold (CPT) test has recently been developed in order to measure the sensory threshold. The CPT test is a useful examination method that allows selective evaluation of various kinds of nerve fibers including A-delta fibers and $\mathrm{C}$ fibers, and measures the current perception threshold, at different frequencies of electric stimulation ${ }^{7}$.

In this study, we investigated the usefulness of therapeutic interventions for neuropathy at the early stage by studying the change in sensory nerve perception with the duration of diabetes mellitus by stimulating A-beta, A-delta and $\mathrm{C}$ fibers, utilizing the characteristics of the current perception threshold (CPT) test. 


\section{SUBJECTS AND METHODS}

The subjects of this study were outpatients who visited D Hospital Department of Neurology with complaints of significant neuropathic symptoms including dullness, numbness and paraesthesia. Those who were chronic drinkers (alcoholic) or those who had a history of taking medications such as antituberculosis or anticancer drugs, muscloskeletal diseases, entrapment neuropathy, myeloradiculopathy and peripheral vascular diseases were excluded from the study. Stimulations of $5 \mathrm{~Hz}, 250 \mathrm{~Hz}$ and $2,000 \mathrm{~Hz}$ were generated with a Neurometer CPT (Neurotron Inc., Baltimore, MD, U.S.A.) and delivered selectively to $\mathrm{C}$ fibers, A-delta fibers and A-beta fibers.

The measurement of the current perception threshold was performed manually. The intensity of the stimulation at $2,000 \mathrm{~Hz}$ increased to $0.01 \mathrm{~mA}$. For cases who had difficulty in sitting or walking, measurements were made while subjects sat in a wheelchair at rest for 5 minutes. Two electrodes coated with gel, used as the conductive medium, were attached to each subject's bilateral DIP (distal interphalangeal) joints of the first toe to search for legs that had a peroneal nerve dysfunction. The Neurometer CPT was used to check peroneal nerve dysfunction in each subject. The current was increased until the patients began to feel the current at the skin where the electrodes were attached, and the perception threshold was measured within $20 \mu \mathrm{A}$ of error by actual stimulations or pseudostimulations to the patients 6 to 10 times at an intensity equal to or of higher than that determined in a single blind study. The perception threshold at $250 \mathrm{~Hz}$ and $5 \mathrm{~Hz}$ was measured in the same manner. For the statistical analysis, the independent t-test was used to investigate differences in the threshold values. The statistical software used was SPSSWIN (ver. 17.0) and a significance level of $\mathrm{p}<0.05$ was chosen.

\section{RESULTS}

There were 224 experimental subjects of whom $64.7 \%$ were men and $35.3 \%$ were women. The men's average age was 42.61 years and the women's was 42.15 years. The average duration of DM was 47.55 months among the men and 58.75 months among the women. The average blood concentration of hemoglobin Alc (HbAlc) was $9.2 \mathrm{mg} / \mathrm{dl}$.

The thresholds values of nerve fibers were measured in order to examine the changes in the degeneration rate between nerve fibers in both the right and left lower limbs. The results show that there were no significant differences in the threshold values of the A-beta and A-delta fibers between the right and the left sides, while the threshold value of $C$ fibers was low $(p<0.05)$. A comparison of the right and the left sides revealed no significant differences in the threshold values of each fiber (Table 1). The threshold values of nerve fibers were also measured in order to examine the differences in degeneration rates among nerve fibers as DM progressed. Starting from the time the disease was first diagnosed, the duration categories were divided as follows: less than 1 year, 1 year to less than 3 years, 3 years to less than 5 years, 5 years to less than 10 years, 10 years or
Table 1. The threshold values of the nerve fibers depending on the measurement parts $(\mu \mathrm{A})$

\begin{tabular}{lccc}
\hline & A-beta & A-delta & C \\
\hline Right* & $12.96 \pm 4.64$ & $12.95 \pm 3.04$ & $10.83 \pm 2.93^{\mathrm{a}}$ \\
Left* & $12.75 \pm 4.87$ & $12.67 \pm 3.66$ & $10.77 \pm 3.61^{\mathrm{a}}$ \\
t-value & .467 & .885 & .216 \\
\hline
\end{tabular}

Mean \pm SD. *: $\mathrm{p}<0.05$.

Post Hoc Test: a: A-beta $=$ A-delta $>C(p<0.05)$.

longer. Variation of the threshold values of the nerve fibers depending on the duration of DM was investigated and the results are as follow. In the case of the right lower limb, the threshold value of A-beta fibers varied with DM duration $(p<0.05)$; the threshold values of the group with 10 years or longer DM duration were higher than those of the groups of other durations according to the post hoc test. The threshold values of A-delta fibers also varied with DM duration $(p<0.05)$; the threshold values of the group of DM duration from 5 years to less than 10 years was not significantly different from that of the group of 10 years or longer duration. However, the threshold value of the 10 years or longer duration group was higher than that of the groups of other durations $(\mathrm{p}<0.05)$ according to the post hoc test. On the other hand, no variation with durations was found in the case of C fibers. The left lower limb did not show significant variation of nerve threshold values with DM durations. Although there were no significant variations of nerve threshold values with duration, according to post hoc test: the threshold value of the 10 years or longer group was higher than those of the 1-year group and 1 year to less than 3 years group in the case of A-beta fibers the threshold value of A-delta fibers in the 10 years or longer group was higher than those of the DM duration groups of less than 1 year, 1 year to less than 3 years, and 3 years to less than 5 years; and the threshold value of $\mathrm{C}$ fibers in the 10 years or longer group was higher than those of the DM duration groups of 1 year to less than 3 years (Table 2).

\section{DISCUSSION}

Neuropathy caused by diabetes mellitus can be a very important disease from the viewpoint of physical therapy since it can increase the risks of fracture and soft tissue injury in falls by reducing patients sensory and motor control ability, and it also accelerates the degeneration of joints and their surrounding tissues, thus causing various kinds of secondary disability ${ }^{8}$. It has been known that symptom and neurological examination, sensory loss, and electrophysiological studies should be included in the diagnosis criteria of neuropathy, and a quantitative sensory test is one of the most meaningful diagnosis methods ${ }^{9)}$. Thus, for this study, we employed a quantitative electrophysiological diagnosis method that evaluates selective peripheral nerve function by measuring the minimum current that is felt by the nerve fiber through the sensory nerve. Neurometer CPT is an instrument that is used in clinical practice for the evaluation of peripheral 
Table 2. Threshold values of the nerve fibers according to the duration of DM $(\mu \mathrm{A})$

\begin{tabular}{lllcc}
\hline & & A-beta & A-delta & C \\
\hline Right & Less than 1 year* & $12.25 \pm 4.16^{\mathrm{c}}$ & $12.52 \pm 2.91^{\mathrm{e}}$ & $10.79 \pm 2.92^{\mathrm{a}, \mathrm{f}}$ \\
& 1 year to less than 3 years* & $11.77 \pm 3.90$ & $12.42 \pm 2.52$ & $9.95 \pm 2.25^{\mathrm{a}}$ \\
& 3 years to less than 5 years & $12.67 \pm 3.82$ & $12.54 \pm 2.41$ & $10.96 \pm 2.49$ \\
& 5 years to less than 10 years* & $13.24 \pm 4.72$ & $13.20 \pm 3.38$ & $10.98 \pm 3.15^{\mathrm{a}}$ \\
& 10 years or longer* & $15.20 \pm 5.73$ & $14.15 \pm 3.42$ & $11.59 \pm 3.40^{\mathrm{a}}$ \\
\hline Left & Less than 1 year* & $11.81 \pm 4.12^{\mathrm{d}}$ & $12.28 \pm 3.38$ & $10.39 \pm 3.38^{\mathrm{a}, \mathrm{f}}$ \\
& 1 year to less than 3 years* & $12.02 \pm 4.26$ & $11.98 \pm 2.82$ & $9.95 \pm 2.47^{\mathrm{a}}$ \\
& 3 years to less than 5 years & $12.79 \pm 4.61$ & $12.83 \pm 3.19$ & $10.67 \pm 2.71$ \\
& 5 years to less than 10 years & $13.18 \pm 5.28$ & $13.10 \pm 4.46$ & $11.39 \pm 4.70$ \\
& 10 years or longer* & $14.51 \pm 5.84$ & $13.41 \pm 4.03$ & $11.56 \pm 3.82^{\mathrm{b}}$ \\
\hline
\end{tabular}

Mean \pm SD. *: $p<0.05$. Post Hoc Test: a: A-beta $=A-d e l t a>C(p<0.05)$. b: A-beta $>C(p<0.05)$. c: less than 1 year $=1$ year to less than 3 years $=3$ years to less than 5 years $=5$ years to less than $10<10$ years or longer $(\mathrm{p}<0.05)$. $\mathrm{d}$ : less than 1 year $=1$ year to less than 3 years $<10$ years or longer $(\mathrm{p}<0.05)$. e: less than 1 year $=1$ year to less than 3 years $=3$ years to less than 5 years $<10$ years or longer $(p<0.05)$. f: 1 year to less than 3 years $<10$ years or longer $(p<0.05)$.

neuropathy and diabetic neuropathy by measuring the sensory threshold. Intraclass correlation coefficients of measurers range between 0.81 and 0.86 , and interclass correlation coefficients among measurers were higher than 0.78 , indicating its high reliability ${ }^{10}$.

The current perception threshold test is designed to test the abnormality of nerve fibers in peripheral nerves by delivering percutaneous current stimulation which can be felt by the subject through the sensory nerve and examining the sine waves at different frequencies ${ }^{7}$. In this study, the variation in threshold values with DM duration of nerve fibers was investigated. The results show that there were significant differences in of the threshold values across all duration groups except the group of 3 years and less than 5 years durations, and that the threshold values of A-beta fibers, and A-delta fibers were significantly higher than that of C fibers. In the case of left lower limb, there was variation among the DM duration groups of less than 1 year, from 1 year to less than 3 years, and 10 years or longer. The threshold values of A-beta fibers and A-delta fibers were higher than that of $\mathrm{C}$ fibers, but they were not significantly different. These results suggest that the degeneration of Abeta fibers and A-delta fibers that have myelin is more rapid than that of $\mathrm{C}$ fibers that are without myelin. The degeneration rate was significantly higher in the DM duration group of 10 years or longer. Therefore, therapeutic interventions should be applied at the early stage of neuropathy.

\section{REFERENCES}

1) Won JY: Epidemiologic study of patients with neuropathic pain in Korea. Dankook University, Dissertation of Doctorate Degree, 2008.

2) Bruyn GW, Garland H: Neuropathies of endocrine origin. Handbook of clinical neurology. Amsterdam : North-Hollo and Publishing Co, 1970, 8:929.

3) Lindsay KW, Bone I, Callander R: Neurology and neurosurgery illustrated, 2nd ed, New York: Churchill Livingstone, 1991, 411-421.

4) Han TR, Baek NJ, Lee SW, et al.: The effect of hyperglycemia on the electrophysiologic changes in diabetic neuropathy. J Korean Academy of Rehabilitation Medicine, 1995, 19: 462-475.

5) Zaslansky R, Yarnitsky D: Clinical applications of quantitative sensory testing. J Neurol Sciences, 1998, 153: 215-238.

6) David Y, Elliot S: Thermal testing: normative data and repeatability for various test algorithm. J Neurol Sciences, 1994, 125: 39-45.

7) Masson EA, Boulton AJ: The Neurometer: validation and comparison with conventional tests for diabetic neuropathy. Diabet Med, 1991, 8: 63-66.

8) Ann M, David E, James M: Evaluation and prevention of diabetic neuropathy. American Academy of Family Physicians, 2005, 71: $2123-$ 2130.

9) Donghue VM, Giurini JM, Rosenblum BI, et al.: Variability in function measurements of three sensory foot nerves in neuropathic diabetic patients. Diabetes Res Clin Pract, 1995, 29: 37-42.

10) Shiiba $Y$, Sakamoto E, Imamura $Y$, et al.: The investigation of practical use of current perception thresholds in the territory of the trigeminal nerve. $\mathrm{J}$ Pain, 2001, 20: 126-132. 